\title{
A comparison of endoscopic and non-endoscopic biliary interven- tion outcomes in patients with prior bariatric surgery
}

\section{다(1)(이우}

\section{Authors}

Amrit K. Kamboj ${ }^{1}$, Victorio Pidlaoan ${ }^{2}$, Mohammad H. Shakhatreh²,3, Alice Hinton ${ }^{4}$, Darwin L. Conwell ${ }^{5}$, Somashekar G. Krishna 5,6

\section{Institutions}

1 Department of Internal Medicine, Mayo Clinic, Rochester, Minnesota, United States

2 Division of Gastroenterology, Hepatology, and Nutrition, The Ohio State University Wexner Medical Center, Columbus, Ohio, United States

3 Section of Gastroenterology and Hepatology, Virginia Tech Carilion School of Medicine and Research Institute, Roanoke, Virginia, United States

4 Division of Biostatistics, College of Public Health, The Ohio State University, Columbus, Ohio, United States

5 Section of Pancreatic Disorders, Division of Gastroenterology, Hepatology and Nutrition, The Ohio State University Wexner Medical Center, Columbus, Ohio, United States

6 Section of Advanced Endoscopy, Division of Gastroenterology, Hepatology, and Nutrition, The Ohio State University Wexner Medical Center, Columbus, Ohio, United States

submitted 20.5.2017

accepted after revision 8.9.2017

\section{Bibliography}

DOI https://doi.org/10.1055/s-0043-121878 |

Endoscopy International Open 2018; 06: E11-E28

(c) Georg Thieme Verlag KG Stuttgart · New York ISSN 2364-3722

\section{Corresponding author}

Somashekar G. Krishna, MD, MPH, Assistant Professor of Medicine, Division of Gastroenterology, Hepatology, and Nutrition, The Ohio State University Wexner Medical Center, 395 West 12th, Avenue, Columbus, OH 43210 Fax: +1-614-293-8518 sgkrishna@gmail.com

\section{ABSTRACT}

Background and study aims Endoscopic biliary intervention (BI) is often difficult to perform in patients with prior bariatric surgery (BRS). We sought to analyze outcomes of patients with prior BRS undergoing endoscopic and nonendoscopic BI.

Patients and methods The Nationwide Inpatient Sample (2007-2011) was reviewed to identify all adult inpatients ( $\geq 18$ years) with a history of BRS undergoing BI. The clinical outcomes of interest were in-patient mortality, length of stay (LOS), and total hospital charges.

Results There were 7,343 patients with prior BRS who underwent Bls where a majority were endoscopic (4,482 vs. $2,861, P<0.01)$. The mean age was $50 \pm 30.8$ years and the majority were females (80.5\%). Gallstone-related disease was the most common indication for $\mathrm{BI}$ and managed more often with primary endoscopic management $(2,146$ vs. $1,132, P<0.01)$. Inpatient mortality was not significantly different between patients undergoing primary endoscopic versus non-endoscopic $\mathrm{BI}(0.2 \%$ vs. $0.7 \%, P=0.2)$. Patients with sepsis were significantly more likely to incur failed primary endoscopic BI (OR 2.74, 95\% CI 1.15, 6.53) and were more likely to be managed with non-endoscopic BI (OR 2.13, $95 \% \mathrm{Cl} 1.3,3.5)$. Primary non-endoscopic $\mathrm{BI}$ and failed endoscopic BI were both associated with longer LOS (by 1.77 days, $P<0.01$ and by 2.17 days, $P<0.01$, respectively) and higher hospitals charges (by $\$ 11,400, P<0.01$ and by $\$ 14,200, P<0.01$, respectively).

Conclusion Primary endoscopic management may be a safe and cost-effective approach for patients with prior BRS who need BI. While primary endoscopic biliary intervention is more common, primary non-endoscopic intervention may be used more often for sepsis.

\section{Introduction}

Morbid obesity continues to rise significantly in the United States and now effects approximately 1 in 15 adults [1,2]. Bariatric surgery (BRS) leads to sustained weight loss and improve- ments in morbidity and mortality [3,4]. Morbidly obese individuals, especially those with a body mass index (BMI) $\geq 40$, have been shown to have an 8-fold higher risk of gallstone formation compared with those with a lower BMI [5]. After BRS, the rapid weight loss often accelerates gallstone formation which may 
predispose to acute cholecystitis, acute pancreatitis, and ascending cholangitis [6]. Despite this risk, prophylactic concomitant cholecsytectomy is not often performed due to its association with increased BRS complications and only a minority of patients developing symptomatic gallstone disease $[7,8]$. Patients with prior BRS often require biliary intervention including endoscopic retrograde cholangio-pancreatography (ERCP) for choledocholithiasis, recurrent pancreatitis, pancreaticobiliary neoplasms, biliary obstruction, and biliary leak [9-11].

Roux-en-Y gastric bypass (RYGB) constitutes the vast majority of BRSs, accounting for approximately $60 \%-70 \%$ of all BRSs [12]. In patients with prior RYGB BRS that require biliary intervention, alteration of the normal foregut anatomy may make access to the native biliary tree very challenging. Conventional ERCP has a success rate of approximately $50 \%$ in patients with prior Whipple resection, with significant higher success rates (84\%) when used for biliary indications [13]. Transgastric access with laparoscopic methods along with endoscopic single and double balloon enteroscopy, have been described with varying methods of success $[9,14]$. In patients with prior RYGB, a laparoscopic-assisted transgastric access with ERCP is recommended for assessment of the duodenum and biliary tree, and evaluation of chronic abdominal pain [15].

To our knowledge, there are no large population-based studies comparing endoscopic (laparoscopic or enteroscopy-assisted ERCP) versus non-endoscopic (percutaneous cholangiography and surgical common bile duct exploration) procedures in patients with prior BRS that require biliary intervention. Thus, our aim was to estimate the prevalence of biliary interventions in patients with prior BRS and to evaluate clinical outcomes comparing endoscopic and non-endoscopic approaches. The clinical outcomes of interest were in-patient mortality, length of stay (LOS), and total hospital charges

\section{Patients and methods}

\section{Data source}

The Nationwide Inpatient Sample (NIS) Healthcare Cost Utilization Project (HCUP), an administrative claims databank, is the largest all-payer inpatient care database in the US [16]. The NIS is a compilation of more than 8 million inpatient admissions from approximately 1000 hospitals (representing about $85 \%$ of all nonfederal hospitals). It is designed to approximate a $20 \%$ stratified probability sample of patients from all nonfederal acute-care hospitals in the US. Discharge weights are provided, which allows extraction of national level estimates from the unweighted database information [16].

The NIS-HCUP database was queried from 2007 to 2011 using the International Classification of Diseases, Ninth Revision, Clinical Modification (ICD-9-CM) diagnosis and procedure for all adult patients ( $\geq 18$ years) with a history of BRS undergoing biliary procedures (ERCP, percutaneous cholangiography (PTC) and surgical common bile duct exploration (CBDE). Due to the limited specificity of ICD-9-CM coding, we were unable to distinguish between laparoscopic, or enteroscopy-assisted ERCP. Therefore, these procedures were grouped into 1 category referred to as endoscopic biliary interventions. Primary endoscopic intervention is defined as the performanance of any endoscopic biliary intervention as the initial procedure for treatment. Primary non-endoscopic intervention is similarly defined as the performance of either PTC or CBDE as the initial therapeutic technique. Failed endoscopic intervention was defined as the performance of a non-endoscopic biliary intervention (PTC or CBDE) within 7 days after a primary endoscopic intervention. A history of prior BRS, etiologic factors for biliary intervention and associated diagnoses, and specific types of biliary intervention were queried by using specific ICD-9-CM codes (Appendix 1).

The Ohio State University Data and Specimen Policy and Human Subjects Research Policy does not require Institutional Review Board approval for population-based public data sets. Per 45 Code of Federal Regulations (CFR 46.101), research using certain publicly available data sets does not involve "human subjects."

\section{Patients and outcomes}

Patient-level variables included age, sex, race, median household income for patient's zip code (quartiles), and insurance status. Race/ethnicity was categorized as White, Black, Hispanic, and others. Insurance status was categorized as Medicare, Medicaid, private insurance, and uninsured/other based on the primary payer listed on the discharge record. Comorbidities for risk adjustment were derived from Agency for Healthcare Research and Quality (AHRQ) comorbidity measures based on the methods by Elixhauser [17]. Patients were given a score of $<3$ or $\geq 3$ based on the number of comorbidities. Hospital-related potential confounders were hospital location (urban vs. rural), hospital bed size (large, medium, small), and hospital teaching status (teaching vs. nonteaching). Hospital bed size was classified as small, medium, or large based on an algorithm developed by HCUP. Hospital region was classified by the US Census Bureau as Northeast, Midwest, South, or West.

Clinical outcomes of interest were inpatient mortality, LOS, and total hospital charges and we compared these in 2 groups of patients with prior BRS: (a) patients requiring primary endoscopic versus non-endoscopic biliary intervention, and (b) patients undergoing failed versus successful endoscopic biliary interventions. The LOS and total hospital charges were collectively referred to as healthcare resource utilization.

\section{Statistical analysis}

Categorical variables and continuous variables were tested for statistical significance with Chi-square tests and $t$ tests, respectively. The mean and standard deviation were calculated for all continuous outcomes and frequency counts and percentages were calculated for all categorical outcomes. Temporal trends were assessed using the Cochrane-Armitage trend test. Univariate predictor variables with a $P$ value $<0.1$ were included in the multivariate analysis. Multivariate linear regression models were fit for continuous outcome variables and multivariate logistic regression models were fit for each dichotomous outcome. All results in the regression model were represented by an odds ratio (OR) and $95 \%$ confidence interval $(\mathrm{Cl})$. All regression models were performed separately. Statistical significance 
Primary diagnosis of $\mathrm{AP}(P<0.001)$

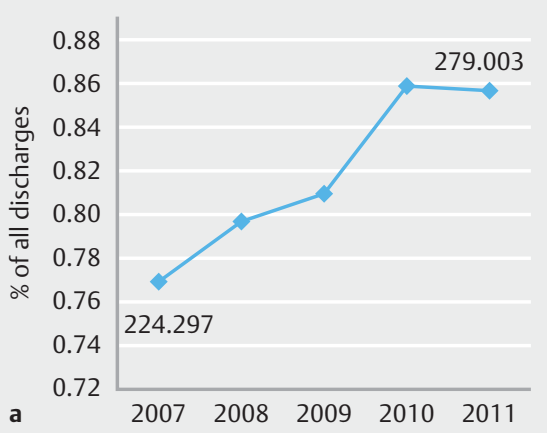

Patients with history of bariatric surgery

$$
(P<0.001)
$$

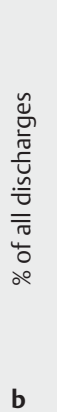

History of bariatric surgery in those with AP $(P<0.001)$

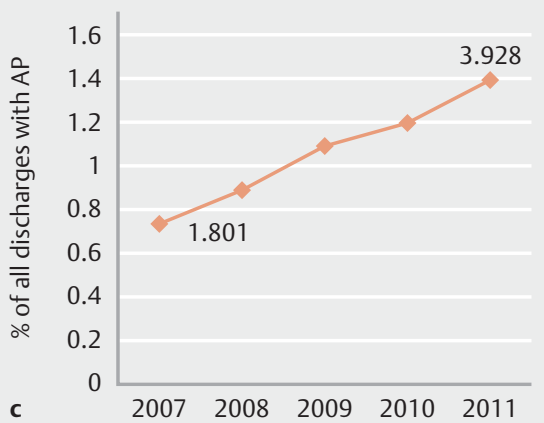

- Fig. 1 Trend analysis of hospitalizations in the Nationwide Inpatient Sample (2007 - 2011). Increasing prevalence of a acute pancreatitis, b patients with history of bariatric surgery, and c history of bariatric surgery in patients admitted with acute pancreatitis.

AP, acute pancreatitis

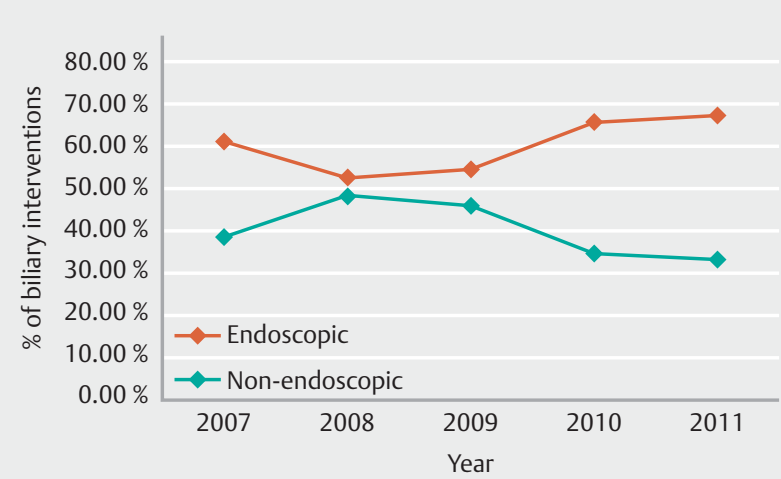

Fig. 2 Trends in endoscopic and non-endoscopic biliary interventions among patients with prior bariatric surgery, Nationwide Inpatient Sample, 2007-2011.

was defined by $P<0.05$. These analyses were performed on weighted data from the NIS database using SAS 9.3 (SAS Institute, Cary, NC) employing appropriate survey procedures to produce national estimates.

Missing data is enlisted in Appendix 2. Race was the variable with the most missing data (9.4\%) since certain states do not document race in discharge information. Other variables had less than $1 \%$ missing data and these were dropped from the final analysis. Imputation was not performed as data was assumed to be missing at random.

\section{Results}

\section{Trends in bariatric surgery, cholecystectomy, and biliary drainage procedures}

The trends in the different types of bariatric surgeries performed in the United States from 2005 to 2011 are illustrated in Appendix 3a. During this time period, the proportion of RYGBs decreased from $81.9 \%$ (97,814 out of 119,382 total BRSs) to $58.3 \%(63,178$ of 108,354$)$, while the proportion of sleeve gastrectomies and gastric band surgeries increased from $18.1 \%$ to $41.7 \%$. The proportion of patients undergoing simultaneous cholecystectomies at the time of BRS decreased from $8.3 \%$ in $2005(9,880$ of 119,382$)$ to $3.4 \%$ in $2011(3,653$ of 108,354), $P<0.001$ (Appendix 3b). Between 2007 and 2011 , there were a total of 988,015 patients discharged with a diagnostic code for history of BRS. The proportion of admissions as well as the total number of patients with prior BRS doubled from 2007 to 2011 (126,872 [0.32\%] to 250,395 [0.65\%], $P<0.001$ ) (> Fig. 1).

For patients with a history of BRS, the proportion of primary endoscopic interventions increased (435 [61.3\%] to 1,346 [66.7\%], $P<0.001$ ) between 2007 and 2011 while the proportion of primary non-endoscopic interventions generally decreased (275 [38.7\%] to 672 [33.3\%], P<0.001) ( Fig. 2).

\section{Patient characteristics and procedure indications}

From 2007 to 2011 , there were $7,343(0.74 \%$ of 988,015$)$ patients with a history of BRS who underwent a biliary intervention. The majority of these patients underwent an endoscopic intervention compared to non-endoscopic interventions $(4,482$ [61\%] vs. 2,861 [39\%] respectively, $P<0.001)(\triangleright \mathrm{Ta}-$ ble 1). Biliary intervention in BRS was more frequent in women and in large urban hospitals. Most procedures (endoscopic or non-endoscopic) were performed within 1 day of hospitalization. A majority of all endoscopic and non-endoscopic interventions were performed for gallstone-related disease $(2,146$ [47.9\%] and 1,132 [39.6\%] respectively). Among patients that underwent non-endoscopic interventions, more required PTC $(1,692[59 \%])$ compared to CBDE (1,169 [41\%]) (Appendix 4).

\section{Endoscopic versus non-endoscopic biliary intervention}

\section{Demographics and hospital variables}

Univariate analysis ( $>$ Table 1 ) revealed that patients who underwent a primary endoscopic biliary intervention were younger, with fewer comorbid conditions, and were treated at teach- 
- Table 1 Demographics, etiological associations, and outcomes of patients with a history of bariatric surgery undergoing endoscopic or non-endoscopic biliary intervention: Comparison of endoscopic versus non-endoscopic (PTC/CBDE) in the Nationwide Inpatient Sample from 2007 to 2011.

\begin{tabular}{|c|c|c|c|c|c|}
\hline \multirow{2}{*}{$\begin{array}{l}\text { Total: } 7,343 \\
\text { Age (mean, SD) }\end{array}$} & \multicolumn{2}{|c|}{$\begin{array}{l}\text { Endoscopic Intervention } \\
n=4,482(\%)\end{array}$} & \multicolumn{2}{|c|}{$\begin{array}{l}\text { Non-endoscopic Intervention } \\
n=2,861(\%)\end{array}$} & \multirow{2}{*}{$\begin{array}{l}P \text { value } \\
0.0039\end{array}$} \\
\hline & 50.11 & 30.76 & 52.14 & 26.25 & \\
\hline Gender & & & & & 0.0003 \\
\hline - Male & 728 & $16.25 \%$ & 705 & $24.66 \%$ & \\
\hline - Female & 3,754 & $83.75 \%$ & 2,156 & $75.34 \%$ & \\
\hline Race & & & & & 0.1189 \\
\hline - White & 3,131 & $76.85 \%$ & 2,105 & $81.48 \%$ & \\
\hline - Black & 402 & $9.86 \%$ & 252 & $9.76 \%$ & \\
\hline - Hispanic & 396 & $9.71 \%$ & 167 & $6.46 \%$ & \\
\hline - Other & 146 & $3.58 \%$ & 60 & $2.31 \%$ & \\
\hline Income (national quartile) & & & & & 0.4937 \\
\hline - 1 & 948 & $21.48 \%$ & 561 & $20.12 \%$ & \\
\hline .2 & 1,145 & $25.95 \%$ & 765 & $27.43 \%$ & \\
\hline .3 & 1,131 & $25.63 \%$ & 793 & $28.41 \%$ & \\
\hline .4 & 1,189 & $26.95 \%$ & 671 & $24.04 \%$ & \\
\hline Type of insurance & & & & & 0.2303 \\
\hline - Medicare & 1,129 & $25.19 \%$ & 833 & $29.11 \%$ & \\
\hline - Medicaid & 297 & $6.62 \%$ & 136 & $4.74 \%$ & \\
\hline - Private & 2,620 & $58.45 \%$ & 1,646 & $57.53 \%$ & \\
\hline - Other & 436 & $9.74 \%$ & 247 & $8.63 \%$ & \\
\hline Hospital location & & & & & 0.0815 \\
\hline - Rural & 167 & $3.78 \%$ & 163 & $5.73 \%$ & \\
\hline - Urban & 4,243 & $96.22 \%$ & 2,678 & $94.27 \%$ & \\
\hline Hospital teaching status & & & & & 0.0218 \\
\hline - Nonteaching & 1,835 & $41.61 \%$ & 1,396 & $49.14 \%$ & \\
\hline - Teaching & 2,575 & $58.39 \%$ & 1,445 & $50.86 \%$ & \\
\hline Hospital size & & & & & 0.8159 \\
\hline - Small & 386 & $8.75 \%$ & 252 & $8.86 \%$ & \\
\hline - Medium & 898 & $20.36 \%$ & 621 & $21.87 \%$ & \\
\hline - Large & 3,126 & $70.88 \%$ & 1,968 & $69.28 \%$ & \\
\hline Hospital region & & & & & 0.0603 \\
\hline - Northeast & 942 & $21.02 \%$ & 535 & $18.69 \%$ & \\
\hline - Midwest & 1,087 & $24.25 \%$ & 540 & $18.88 \%$ & \\
\hline - South & 1,321 & $29.48 \%$ & 1,057 & $36.96 \%$ & \\
\hline - West & 1,132 & $25.25 \%$ & 729 & $25.48 \%$ & \\
\hline Weekend admission & & & & & 0.4549 \\
\hline - No & 3,717 & $82.94 \%$ & 2,331 & $81.46 \%$ & \\
\hline - Yes & 765 & $17.06 \%$ & 530 & $18.54 \%$ & \\
\hline Elixhauser comorbidity Index & & & & & $<0.0001$ \\
\hline
\end{tabular}


- Table 1 (Continuation)

\begin{tabular}{|c|c|c|c|c|c|}
\hline \multirow{2}{*}{$\begin{array}{l}\text { Total: } 7,343 \\
\text { - }<3\end{array}$} & \multicolumn{2}{|c|}{$\begin{array}{l}\text { Endoscopic Intervention } \\
n=4,482(\%)\end{array}$} & \multicolumn{2}{|c|}{$\begin{array}{l}\text { Non-endoscopic Intervention } \\
n=2,861 \text { (\%) }\end{array}$} & \multirow[t]{2}{*}{$P$ value } \\
\hline & 2,860 & $63.82 \%$ & 1,508 & $52.71 \%$ & \\
\hline - $\geq 3$ & 1,622 & $36.18 \%$ & 1,353 & $47.29 \%$ & \\
\hline \multicolumn{6}{|l|}{ Etiology } \\
\hline Gallstone related & 2,146 & $47.88 \%$ & 1,132 & $39.57 \%$ & 0.0023 \\
\hline Pancreaticobiliary neoplasm & 81 & $1.80 \%$ & 197 & $6.89 \%$ & $<0.0001$ \\
\hline Disease of bile duct & 223 & $4.97 \%$ & 96 & $3.34 \%$ & 0.1383 \\
\hline Bile leak, bile duct injury, biliary peritonitis & 253 & $5.63 \%$ & 309 & $10.81 \%$ & 0.0008 \\
\hline Bile duct obstruction and jaundice NOS & 262 & $5.84 \%$ & 186 & $6.51 \%$ & 0.6299 \\
\hline Stent-related (changes, others) & 134 & $2.99 \%$ & 53 & $1.86 \%$ & 0.1597 \\
\hline Chronic pancreatitis & 54 & $1.21 \%$ & $\mathrm{a}$ & $0.00 \%$ & - \\
\hline Sphincter of oddi dysfunction & 22 & $0.50 \%$ & $\mathrm{a}$ & $0.11 \%$ & 0.1812 \\
\hline Abdominal pain & 41 & $0.92 \%$ & $\mathrm{a}$ & $0.00 \%$ & - \\
\hline Time to PTC/CBDE or ERCP & & & & & 0.2628 \\
\hline - $<0$ to 1 day & 2,294 & $51.19 \%$ & 1,571 & $54.90 \%$ & \\
\hline - $2-4$ days & 1,593 & $35.53 \%$ & 890 & $31.11 \%$ & \\
\hline - 5-10 days & 595 & $13.27 \%$ & 400 & $13.99 \%$ & \\
\hline Cholecystectomy & 1,697 & $37.86 \%$ & 1,135 & $39.66 \%$ & 0.5294 \\
\hline Associated diagnoses & \multicolumn{2}{|c|}{ (DX1-DX25) } & & & \\
\hline Sepsis & 184 & $4.10 \%$ & 303 & $10.58 \%$ & $<0.0001$ \\
\hline Acute pancreatitis & 1,146 & $25.57 \%$ & 360 & $12.57 \%$ & $<0.0001$ \\
\hline Cholangitis & 532 & $11.87 \%$ & 387 & $13.54 \%$ & 0.3403 \\
\hline \multicolumn{6}{|l|}{ Outcome } \\
\hline Death & a & $0.22 \%$ & 20 & $0.69 \%$ & 0.2181 \\
\hline Length of stay $\geq 7$ days & 1,215 & $27.12 \%$ & 1,352 & $47.25 \%$ & $<0.0001$ \\
\hline Length of stay (mean, SD) & 5.31 & 9.46 & 7.40 & 11.69 & $<0.0001$ \\
\hline Total charges (mean, SD) & 50,664 & 92,279 & 64,349 & 125,999 & $<0.0001$ \\
\hline
\end{tabular}

ing hospitals compared to those requiring primary non-endoscopic interventions. Gallstone-related disease was associated with more frequent primary endoscopic management while pancreaticobiliary neoplasms and bile duct injury were associated with primary non-endoscopic management.

\section{Presence of associated emergent conditions}

Acute pancreatitis (AP), as an associated diagnosis, was more frequent in patients requiring an endoscopic approach; however, sepsis, as an associated diagnosis, was more frequent in patients undergoing non-endoscopic biliary interventions ( $\triangleright$ Table 1). Multivariate analysis adjusting for demographics, hospital factors, and etiologies confirmed these findings. Sepsis was more than 2 times more likely to be associated with patients requiring non-endoscopic intervention (OR 2.13, $95 \% \mathrm{Cl}$ $1.30,3.50, P=0.003)$. On the contrary, AP was more than 2 times more frequently associated with patients undergoing an endoscopic approach $(\mathrm{OR}=2.44,95 \% \mathrm{Cl} 0.30,0.56, P<0.001)$.

\section{Mortality and health care resource utilization}

The overall in-hospital mortality rate for patients with prior BRS undergoing biliary intervention was $0.41 \%$ (30 of 7,343 patients). Inpatient mortality was not significantly different between patients undergoing primary endoscopic versus primary non-endoscopic procedures $(0.22 \%$ vs. $0.69 \%, P=0.2)$ ( $\triangleright$ Table 1). However, non-endoscopic interventions were associated 
- Table 2 Multivariate linear regression model for healthcare utilization in patients with a history of bariatric surgery undergoing biliary intervention, Nationwide Inpatient Sample, 2007-2011.

\begin{tabular}{|c|c|c|c|c|c|c|}
\hline & \multicolumn{3}{|c|}{ Length of stay } & \multicolumn{3}{|c|}{ Total charges } \\
\hline & Days & $95 \% \mathrm{Cl}$ & $P$ value & $\$$ & $95 \% \mathrm{Cl}$ & $P$ value \\
\hline Primary procedure & & & $<0.0001$ & & & $<0.0001$ \\
\hline - ERCP & Reference & & & Reference & & \\
\hline - CBDE/PTC & 1.77 & $(1.32,2.21)$ & & 11,453 & $(5,811,17,095)$ & \\
\hline Age & 0.01 & $(-0.01,0.02)$ & 0.423 & -78 & $(-225,69)$ & 0.3001 \\
\hline Gender & & & & & 0.7499 & 0.0316 \\
\hline - Male & Reference & & & Reference & & \\
\hline - Female & -0.09 & $(-0.61,0.44)$ & & $-6,460$ & $(-12,351,-569)$ & \\
\hline Hospital location & & & 0.0854 & & & $<0.0001$ \\
\hline - Rural & Reference & & & Reference & & \\
\hline - Urban & 0.64 & $(-0.09,1.37)$ & & 19,625 & $(12,775,26,475)$ & \\
\hline Hospital teaching status & & & 0.9229 & & & 0.7444 \\
\hline - Nonteaching & Reference & & & Reference & & \\
\hline - Teaching & 0.03 & $(-0.54,0.59)$ & & $-1,177$ & $(-8,254,5,901)$ & \\
\hline Hospital region & & & 0.9181 & & & 0.0001 \\
\hline - Northeast & Reference & & & Reference & & \\
\hline - West & -0.16 & $(-0.92,0.59)$ & & 14,764 & $(3,652,25,876)$ & \\
\hline - South & 0.04 & $(-0.56,0.64)$ & & $-2,088$ & $(-11,703,7,526)$ & \\
\hline - Midwest & 0.09 & $(-0.54,0.72)$ & & $-6,548$ & $(-16,804,3,708)$ & \\
\hline Elixhauser comorbidity Index & & & $<0.0001$ & & & $<0.0001$ \\
\hline . $<3$ & Reference & & & Reference & & \\
\hline - $\geq 3$ & 1.27 & $(0.79,1.75)$ & & 10,826 & $(5,559,16,092)$ & \\
\hline Gallstone related & -0.79 & $(-1.21,-0.37)$ & 0.0002 & $-3,651$ & $(-8,654,1,351)$ & 0.1525 \\
\hline Pancreaticobiliary neoplasm & 1.55 & $(-0.09,3.19)$ & 0.0639 & 3,699 & $(-9,976,17,373)$ & 0.5959 \\
\hline $\begin{array}{l}\text { Bile leak, bile duct injury, biliary } \\
\text { peritonitis }\end{array}$ & 0.79 & $(-0.37,1.94)$ & 0.1806 & 5,833 & $(-6,525,18,191)$ & 0.3547 \\
\hline Chronic pancreatitis & -1.35 & $(-1.89,-0.80)$ & $<0.0001$ & $-18,484$ & $(-38,533,1,565)$ & 0.0707 \\
\hline Time to PTC/CBDE or ERCP & & & $<0.0001$ & & & $<0.0001$ \\
\hline - $<0$ to 1 day & Reference & & & Reference & & \\
\hline - $2-4$ days & 1.72 & $(1.27,2.16)$ & & 12,004 & $(7,454,16,553)$ & \\
\hline - 5-10 days & 6.29 & $(5.45,7.14)$ & & 43,740 & $(33,291,54,189)$ & \\
\hline
\end{tabular}

CBDE, common bile duct exploration; ERCP, endoscopy retrograde cholangiopancreatography; PTC, percutaneous transhepatic cholangiography;

with a longer length of hospital stay and greater total hospital charges. More specifically, patients with a primary non-endoscopic intervention stayed $1.77(95 \% \mathrm{Cl} 1.32,2.21, P<0.001)$ days longer and were charged $\$ 11,453$ (95\% Cl 5,811, 17,095, $P<0.001)$ more than those with a primary endoscopic intervention ( $\triangleright$ Table 2). Notably, patients who underwent any biliary intervention within 1 day of hospitalization accounted for significantly lower health care resource utilization ( $\triangleright$ Table 2 ).

\section{Successful versus failed endoscopic interventions}

Demographics and hospital variables

A total of 4,482 patients with history of BRS underwent primary endoscopic interventions. Procedure success and failure rates were $88.3 \%(n=3,956)$ and $11.7 \%(n=526)$ respectively, $P<$ 0.001 . Univariate analysis ( $\triangleright$ Table 3 ) revealed that patients who had successful procedures were younger compared to 
those who had a failed procedure. Failed procedures were associated with more frequent cholecystectomies compared to successful procedures ( $47.1 \%$ and $36.6 \%$ respectively, $P=0.04$ ).

\section{Presence of associated emergent conditions}

Acute pancreatitis was an associated diagnosis found more frequently in patients with successful endoscopic interventions while sepsis was an associated diagnosis more frequent in failed interventions ( $\triangleright$ Table 3 ). Specifically, sepsis was more than 2.7 times more likely to be associated with failed endoscopic interventions (OR 2.74, $95 \% \mathrm{Cl} 1.15,6.53, P=0.02$ ) compared to successful interventions.

\section{Mortality and health care resource utilization}

There was no documented death for patients with failed endoscopic interventions and all 10 deaths in the endoscopic intervention group occurred in patients with successful procedures. Failed endoscopic interventions accounted for greater healthcare resource utilization (longer LOS and greater total charges). Specifically, failed endoscopic interventions necessitated 2.17 $(95 \% \mathrm{Cl} 1.79,3.33, P<0.001)$ additional days of stay and $\$ 14,214$ (95\% Cl 3,749, 24,679, $P=0.008$ ) more than successful interventions ( $\triangleright$ Table 4 ). Patients who underwent either successful or failed endoscopic intervention within 1 day of hospitalization accounted for significantly lower health care resource utilization, $P<0.001$.

\section{Discussion}

In this population-based study analysis of all biliary interventions in hospitalized patients with prior BRS from 2007 to 2011, we have demonstrated that gallstone disease is the most common indication for biliary intervention. To our knowledge, this is is the most comprehensive population-based study comparing outcomes of endoscopic versus non-endoscopic interventions in patients with biliary disease and a prior history of BRS. For all patients with BRS needing biliary interventions, a majority underwent endoscopic (ERCP or enteroscopy-assisted or laparscopic-assisted ERCP) guided procedure. Patients with sepsis were significantly more likely to incur failed primary endoscopic BI and were more likely to be managed with nonendoscopic BI. Although there was no difference in inpatient mortality comparing different types of biliary intervention, primary non-endoscopic interventions were associated with increased healthcare resource utilization. Failed endoscopic interventions did not result in greater inpatient mortality but did account for increased healthcare resource utilization.

Our study highlights recent trends in BRS including a steady decrease in RYGBs with a concomittant increase in sleeve gastrectomies; which is consistent with prior studies [18]. Multiple studies have illustrated the increase in prevalence of gallstones with rapid weight loss following BRS, although to varying degrees $[5,19]$. Even though the total number of patients with a history of BRS doubled during the study period, the proportion of patients undergoing simultaneous cholecystectomies at the time of BRS decreased by approximately $60 \%$. Another study analyzing NIS trends during BRS illustrated that the proportion of patients undergoing concomitant cholecystectomy decreased from $26.3 \%$ in 2001 to $3.7 \%$ in 2008 [8]. Concomitant cholecystectomy during gastric bypass surgery is no longer routine practice because operative time, postoperative hospital stay, and postoperative morbidity and mortality are higher with prophylactic cholecystectomy [20]. Several studies have indicated its use only in cases of symptomatic gallbladder disease, particularly cholelithiasis [21].

Among patients who required biliary intervention, the majority underwent primary endoscopic intervention compared to non-endoscopic intervention. The endoscopic intervention failure rate was $12 \%$. However, the database does not differentiate between the 3 major types of bariatric surgeries and endoscopic biliary intervention is more difficult in patients with RYGB anatomy. Furthermore, prior studies have demonstrated that $60 \%$ to $70 \%$ of all BRS patients had RYGB; thus, we can project that the failure rate of endoscopic biliary intervention in patients with RYGB anatomy would be $17 \%$ to $20 \%$ [18]. This failure rate is comparable to prior literature. With the steady decrease in RYGBs along with an increase in sleeve gastrectomies, the success rate of endoscopic biliary interventions may rise in the future, as the latter procedure, in theory, allows for easier access to the papilla compared to the former. In long limb surgical bypass patients with suspected pancreatobiliary diseases, ERCP was successful in $63 \%$ of patients, and specifically in $88 \%$ when the papilla was reached [22]. Common reasons for ERCP failure include afferent limb entered but papilla not reached, cannulation failure, afferent limb angulation, and jejunojejunostomy not identified [22]. Thus, a safe and effective alternative to these modalities in RYGB patients is laparoscopic transgastric endoscopy [23,24]. Laparoscopic-assisted ERCP has been shown to be superior than balloon enteroscopy assisted ERCP with a $100 \%$ rate of papilla identification, cannulation rate, and therapeutic success [25]. However, this procedure should be preferred in patients with Roux + biliopancreatic limb (from ligament of Treitz to jejunojejunal anastomosis) of $150 \mathrm{~cm}$ or longer while those with a limb length less than 150 cm should be offered deep enteroscopy-assisted ERCP first [25].

In this study, overall mortality with either endoscopic or non-endoscopic biliary intervention was $0.41 \%$ and there was no difference in mortality between the 2 groups. Notably, primary non-endoscopic and failed endoscopic interventions accounted for increased healthcare resource utilization. A cohort study utilizing administrative data demonstrated that in all patients presenting with biliary emergencies, failed ERCP and open cholecystectomy were associated with increased mortality and increased healthcare resource utilization [26]. Another retrospective analysis showed that failed ERCP prolongs hospital stays and increases costs of hospitalization [27]. The sickest patients in our study (those with sepsis) required primary or secondary non-endoscopic intervention and hence contributed to increased healthcare resource utilization. Failed ERCP may be a marker for sepsis resulting from delayed biliary decompression leading to increased need for hospital-based interventions. This association was demonstrated in this study where patients with sepsis were managed with non-endoscopic 
- Table 3 Demographics, etiological associations, and outcomes of patients with a history of bariatric surgery undergoing endoscopic intervention: Comparison of endoscopic intervention success in the Nationwide Inpatient database from 2007 to 2011.

\begin{tabular}{|c|c|c|c|c|c|}
\hline \multirow{2}{*}{$\begin{array}{l}\text { Total: } \mathbf{4 , 4 8 2} \\
\text { Age (mean, SD) }\end{array}$} & \multicolumn{2}{|c|}{$\begin{array}{l}\text { Successful endoscopic intervention } \\
n=3,956(\%)\end{array}$} & \multicolumn{2}{|c|}{$\begin{array}{l}\text { Failed endoscopic intervention } \\
n=526(\%)\end{array}$} & \multirow{2}{*}{$\begin{array}{l}\boldsymbol{P} \text { value } \\
0.0132\end{array}$} \\
\hline & 49.68 & 13.69 & 53.35 & 14.91 & \\
\hline Gender & & & & & 0.6979 \\
\hline - Male & 650 & $16.42 \%$ & 79 & $14.97 \%$ & \\
\hline - Female & 3,306 & $83.58 \%$ & 447 & $85.03 \%$ & \\
\hline Race & & & & & 0.0901 \\
\hline - White & 2,744 & $76.14 \%$ & 387 & $82.26 \%$ & \\
\hline - Black & 351 & $9.75 \%$ & 50 & $10.70 \%$ & \\
\hline - Hispanic & 368 & $10.21 \%$ & 28 & $5.89 \%$ & \\
\hline - Other & 140 & $3.89 \%$ & a & $1.15 \%$ & \\
\hline Income (national quartile) & & & & & 0.2689 \\
\hline . 1 & 823 & $21.11 \%$ & 125 & $24.20 \%$ & \\
\hline . 2 & 984 & $25.26 \%$ & 161 & $31.12 \%$ & \\
\hline .3 & 1,006 & $25.81 \%$ & 125 & $24.29 \%$ & \\
\hline .4 & 1,084 & $27.82 \%$ & 105 & $20.39 \%$ & \\
\hline Type of insurance & & & & & 0.5429 \\
\hline - Medicare & 967 & $24.43 \%$ & 162 & $30.85 \%$ & \\
\hline - Medicaid & 262 & $6.61 \%$ & 35 & $6.71 \%$ & \\
\hline - Private & 2,342 & $59.22 \%$ & 277 & $52.71 \%$ & \\
\hline - Other & 385 & $9.74 \%$ & 51 & $9.74 \%$ & \\
\hline Hospital location & & & & & 0.7970 \\
\hline - Rural & 149 & $3.84 \%$ & 18 & $3.36 \%$ & \\
\hline - Urban & 3,740 & $96.16 \%$ & 503 & $96.64 \%$ & \\
\hline Hospital teaching status & & & & & 0.7989 \\
\hline - Nonteaching & 1,624 & $41.76 \%$ & 211 & $40.44 \%$ & \\
\hline - Teaching & 2,265 & $58.24 \%$ & 310 & $59.56 \%$ & \\
\hline Hospital size & & & & & 0.0486 \\
\hline - Small & 354 & $9.10 \%$ & 32 & $6.16 \%$ & \\
\hline - Medium & 829 & $21.31 \%$ & 69 & $13.25 \%$ & \\
\hline - Large & 2,706 & $69.59 \%$ & 420 & $80.59 \%$ & \\
\hline Hospital region & & & & & 0.6458 \\
\hline - Northeast & 851 & $21.52 \%$ & 91 & $17.28 \%$ & \\
\hline - Midwest & 969 & $24.49 \%$ & 118 & $22.45 \%$ & \\
\hline - South & 1,153 & $29.14 \%$ & 168 & $32.00 \%$ & \\
\hline - West & 983 & $24.85 \%$ & 149 & $28.27 \%$ & \\
\hline Weekend admission & & & & & 0.1778 \\
\hline - No & 3,261 & $82.43 \%$ & 457 & $86.81 \%$ & \\
\hline - Yes & 695 & $17.57 \%$ & 69 & $13.19 \%$ & \\
\hline
\end{tabular}


- Table 3 (Continuation)

\begin{tabular}{|c|c|c|c|c|c|}
\hline Total: 4,482 & \multicolumn{2}{|c|}{$\begin{array}{l}\text { Successful endoscopic intervention } \\
n=3,956(\%)\end{array}$} & \multicolumn{2}{|c|}{$\begin{array}{l}\text { Failed endoscopic intervention } \\
n=526(\%)\end{array}$} & \multirow{2}{*}{$\begin{array}{l}P \text { value } \\
0.2182\end{array}$} \\
\hline Elixhauser comorbidity Index & & & & & \\
\hline . $<3$ & 2,553 & $64.53 \%$ & 308 & $58.48 \%$ & \\
\hline . $\geq 3$ & 1,403 & $35.47 \%$ & 218 & $41.52 \%$ & \\
\hline \multicolumn{6}{|l|}{ ETIOLOGY } \\
\hline Gallstone related & 1,863 & $47.11 \%$ & 282 & $53.69 \%$ & 0.2901 \\
\hline Pancreaticobiliary neoplasm & 76 & $1.93 \%$ & a & $0.86 \%$ & 0.2917 \\
\hline Disease of bile duct & 207 & $5.23 \%$ & 16 & $3.02 \%$ & 0.2783 \\
\hline Bile leak, bile duct injury, biliary peritonitis & 202 & $5.11 \%$ & 50 & $9.54 \%$ & 0.1312 \\
\hline Bile duct obstruction and jaundice NOS & 218 & $5.52 \%$ & 43 & $8.25 \%$ & 0.3393 \\
\hline Stent related (changes, others) & 125 & $3.16 \%$ & a & $1.72 \%$ & 0.2898 \\
\hline Chronic pancreatitis & 54 & $1.38 \%$ & a & $0.00 \%$ & - \\
\hline Sphincter of Oddi dysfunction & 22 & $0.56 \%$ & a & $0.00 \%$ & - \\
\hline Abdominal pain & 36 & $0.91 \%$ & a & $0.97 \%$ & 0.9534 \\
\hline Time to ERCP & & & & & 0.4979 \\
\hline - $<0$ to 1 day & 2,029 & $51.29 \%$ & 266 & $50.46 \%$ & \\
\hline - $2-4$ days & 1,419 & $35.88 \%$ & 173 & $32.90 \%$ & \\
\hline - 5-10 days & 507 & $12.83 \%$ & 88 & $16.63 \%$ & \\
\hline Cholecystectomy & 1,449 & $36.63 \%$ & 248 & $47.08 \%$ & 0.0401 \\
\hline Associated diagnoses & \multicolumn{2}{|c|}{ (DX1-DX25) } & & & \\
\hline Sepsis & 130 & $3.27 \%$ & 54 & $10.30 \%$ & 0.0286 \\
\hline Acute pancreatitis & 1,047 & $26.47 \%$ & 99 & $18.79 \%$ & 0.0429 \\
\hline Cholangitis & 444 & $11.23 \%$ & 88 & $16.69 \%$ & 0.1715 \\
\hline \multicolumn{6}{|l|}{ Outcome } \\
\hline Death & a & $0.25 \%$ & a & $0.00 \%$ & - \\
\hline Length of stay $\geq 7$ days & 964 & $24.37 \%$ & 251 & $47.80 \%$ & $<0.0001$ \\
\hline Length of stay (mean, SD) & 5.03 & 4.03 & 7.36 & 5.32 & $<0.0001$ \\
\hline Total charges (mean, SD) & 48,981 & 41,197 & 63,200 & 45,989 & 0.0040 \\
\hline \multicolumn{6}{|c|}{$\begin{array}{l}\text { a) The cell's value is not displayed. As per data agreements with AHRQ, researchers cannot report any statistics where the number of observations in any given cell o } \\
\text { analyzed data is } \leq 10 \text {. } \\
\text { ERCP, endoscopy retrograde cholangiopancreatography }\end{array}$} \\
\hline
\end{tabular}

interventions and more likely to incur failed ERCP. However, difficulties in timing an endoscopic intervention appropriately may explain why patients with sepsis were more often managed with non-endoscopic interventins. A statistically significant mortality difference may have not been seen due to the relatively low death rate and improvements in the management of sepsis [28, 29].

Early biliary intervention in patients with a history of BRS is critical when clinically indicated as patients who underwent biliary intervention within 1 day of hospitalization accounted for significantly lower health care resource utilization. The literature on the timing of endoscopic intervention after hospital admission in patients with BRS remains limited; however, early ERCP has been described in the non-bariatric population. A prospective multicenter study analyzing early ERCP (within 72 hours) versus conservative treatment for acute non-obstructive biliary pancreatitis found that early ERCP was not beneficial in these patients [30]. Other systematic reviews have also found that early ERCP does not effect mortality and complications in patients with acute gallstone pancreatitis compared to conser- 
- Table 4 Multivariate linear regression model for healthcare utilization in patients with a history of bariatric surgery undergoing endoscopic biliary intervention, Nationwide Inpatient Sample, 2007-2011.

\begin{tabular}{|c|c|c|c|c|c|c|}
\hline & \multicolumn{3}{|c|}{ Length of stay } & \multicolumn{3}{|c|}{ Total charges } \\
\hline & Days & $95 \% \mathrm{Cl}$ & $P$ value & $\$$ & $95 \% \mathrm{Cl}$ & $P$ value \\
\hline Endoscopic intervention & & & $<0.0001$ & & & 0.0078 \\
\hline - Successful & Reference & & & Reference & & \\
\hline - Failed & 2.17 & $(1.18,3.16)$ & & 14,214 & $(3,749,24,679)$ & \\
\hline Age & 0.01 & $(-0.01,0.03)$ & 0.1937 & 149 & $(-11,309)$ & 0.0688 \\
\hline Race & & & 0.7441 & & & 0.0834 \\
\hline - White & Reference & & & Reference & & \\
\hline - Black & -0.02 & $(-0.75,0.72)$ & & 4,450 & $(-5,146,14,046)$ & \\
\hline - Hispanic & -0.28 & $(-0.93,0.37)$ & & 8,751 & $(1,822,15,680)$ & \\
\hline - Other & 0.37 & $(-0.92,1.65)$ & & $-1,142$ & $(-14,208,11,924)$ & \\
\hline Hospital size & & & 0.163 & & & 0.4041 \\
\hline - Small & Reference & & & Reference & & \\
\hline - Medium & 0.14 & $(-0.46,0.74)$ & & 4,017 & $(-8,061,16,096)$ & \\
\hline - Large & 0.49 & $(-0.06,1.05)$ & & 6,322 & $(-3,111,15,756)$ & \\
\hline Chronic pancreatitis & -0.18 & $(-0.86,0.50)$ & 0.611 & $-13,961$ & $(-33,873,5,950)$ & 0.1692 \\
\hline Time to ERCP & & & $<0.0001$ & & & $<0.0001$ \\
\hline - $<0$ to 1 day & Reference & & & Reference & & \\
\hline - 2-4 days & 1.87 & $(1.42,2.33)$ & & 14,835 & $(9,635,20,034)$ & \\
\hline - 5-10 days & 7.16 & $(6.11,8.22)$ & & 39,107 & $(28,564,49,649)$ & \\
\hline
\end{tabular}

vative treatment $[31,32]$. However, in patients with co-exisiting cholangitis and biliary obstruction, early ERCP significantly reduced mortality and complications [31].

Given changing trends in prevalence of different types of BRS during the study period, we performed a univariate and multivariate sensitivity analysis of the study time period. Specifically, we dichotomized the study period into 2007-2008 and 2009-2011. Prior studies have demonstrated that gallstonerelated problems are typically seen within 1 to 2 years of bariatric surgery [33]. In one study, the mean follow-up time to cholecystectomy for symptomatic gallstone disease after BRS was 21.5 months [33]. Accordingly, we dichotomized the years into these 2 categories as our trend analysis demonstrated that the decrease in RYGB and increase in sleeve gastrectomies was after 2008. Endoscopic biliary intervention was significantly more frequent in the later time period, $2009-2011$ (62.9\%), compared to 2007-2008 (55.7\%) $(P=0.05)$. However, we found that there were higher total charges (by $\$ 6,378, P=$ 0.03 ) in 2009-2011 (not adjusted for inflation) and no differences in the length of stay (by 0.07 days, $P=0.77$ ) (Appendix 5). Moreover, there were no differences between successful and failed interventions during the 2 time periods. While successful endoscopic interventions were more frequent in 2009-2011
(89.2\%) compared to $2007-2008$ (85.1\%), this difference was not statistically significant $(P=0.13)$, and this did not impact health care utilization (Appendix 6).

As with all administrative databases, coding errors represent a potential limitation of the present study. In the absence of a national bariatric surgery registry, NIS represents a great data source for different types of BRS given its sophisticated sampling design and large number of observations. However, the code for prior-BRS (v45.86) is a v-code, which unfortunately does not detail the various types of bariatric surgeries. However, based on prior studies, we can project that $60 \%$ to $70 \%$ of all BRS patients had RYGB anatomy [18]. Moreover, the ICD9 code for BRS has been utilized in other studies in the literature $[12,34]$. In addition to the potential for miscoding, some unique features of the NIS database should be recognized. First, this study was unable to differentiate between endoscopic and laparoscopic-guided ERCP due to a lack of specificity in the ICD9 codes. Second, the presence of an ICD-9 code for gallstones only proves an association but doesn't convey causality. Third, this database is unable to differentiate distinctive patients, and therefore patients with recurrent biliary interventions could be represented multiple times. The influence of this on the current results is uncertain but expected to be of small magnitude con- 
sidering the statistically large sample size. Lastly, the NIS database cannot account for unobserved characteristics that may influence an intervention, complication, or outcome, so inferring "causality" from observed associations is not valid.

\section{Conclusion}

In conclusion, rates of obesity and prevalence of BRS for morbidly obese patients are increasing. In the vast majority of patients with BRS, concurrent prophylactic cholecystectomy is not performed. As a result, the most common indication for biliary intervention in this population is gallbladder-related disease. While primary endoscopic biliary intervention is more common, primary non-endoscopic intervention may be used more often for sepsis. Future research on improving success rates of endoscopic biliary intervention is prudent to reduce healthcare resource utilization.

\section{Competing interests}

None

\section{References}

[1] Hedley AA, Ogden CL, Johnson CL et al. Prevalence of overweight and obesity among US children, adolescents, and adults, 1999-2002. JAMA 2004; 291: $2847-2850$

[2] Sturm R, Hattori A. Morbid obesity rates continue to rise rapidly in the United States. Int J Obes 2013; 37: 889-891

[3] Christou NV, Sampalis JS, Liberman M et al. Surgery decreases longterm mortality, morbidity, and health care use in morbidly obese patients. Ann Surg 2004; 240: 416 - 423

[4] Colquitt JL, Pickett K, Loveman E et al. Surgery for weight loss in adults. Cochrane Database Syst Rev 2014; 8: CD003641

[5] Iglezias Brandao deOliveira C, Adami ChaimE, da Silva BB. Impact of rapid weight reduction on risk of cholelithiasis after bariatric surgery. Obes Surg 2003; 13: 625-628

[6] Shiffman ML, Sugerman HJ, Kellum JM et al. Gallstone formation after rapid weight loss: a prospective study in patients undergoing gastric bypass surgery for treatment of morbid obesity. Am J Gastroenterol 1991; 86: 1000 - 1005

[7] Mason EE, Renquist KE. Gallbladder management in obesity surgery. Obes Surg 2002; 12: $222-229$

[8] Worni M, Guller U, Shah A et al. Cholecystectomy concomitant with laparoscopic gastric bypass: a trend analysis of the nationwide inpatient sample from 2001 to 2008. Obes Surg 2012; 22: 220-229

[9] Choi EK, Chiorean MV, Cote GA et al. ERCP via gastrostomy vs. double balloon enteroscopy in patients with prior bariatric Roux-en-Y gastric bypass surgery. Surg Endosc 2013; 27: 2894-2899

[10] Bowman E, Greenberg J, Garren M et al. Laparoscopic-assisted ERCP and EUS in patients with prior Roux-en-Y gastric bypass surgery: a dual-center case series experience. Surg Endosc 2016; 30: 4647 4652

[11] Saleem A, Levy MJ, Petersen BT et al. Laparoscopic assisted ERCP in Roux-en-Y gastric bypass (RYGB) surgery patients. J Gastrointest Surg 2012; 16: $203-208$
[12] Krishna SG, Behzadi ], Hinton A et al. Effects of bariatric surgery on outcomes of patients with acute pancreatitis. Clin Gastroenterol Hepatol 2016; 14: $1001-1010$

[13] Chahal P, Baron TH, Topazian MD et al. Endoscopic retrograde cholangiopancreatography in post-Whipple patients. Endoscopy 2006; 38: $1241-1245$

[14] Maaser C, Lenze F, Bokemeyer M et al. Double balloon enteroscopy: a useful tool for diagnostic and therapeutic procedures in the pancreaticobiliary system. Am J Gastroenterol 2008; 103: 894-900

[15] Nguyen NT, Hinojosa MW, Slone J et al. Laparoscopic transgastric access to the biliary tree after Roux-en-Y gastric bypass. Obes Surg 2007; 17: $416-419$

[16] (NIS) HNIS. Healthcare Cost and Utilization Project (HCUP). Agency for Healthcare Research and Quality, Rockville, MD 2007, 2008, 2009, 2010, 2011.

[17] Elixhauser A, Steiner C, Harris DR et al. Comorbidity measures for use with administrative data. Med Care 1998; 36: 8-27

[18] Nguyen NT, Nguyen B, Gebhart A et al. Changes in the makeup of bariatric surgery: a national increase in use of laparoscopic sleeve gastrectomy. J Am Coll Surg 2013; 216: 252 - 257

[19] Kiewiet RM, Durian MF, van Leersum M et al. Gallstone formation after weight loss following gastric banding in morbidly obese Dutch patients. Obes Surg 2006; 16: $592-596$

[20] Warschkow R, Tarantino I, Ukegjini K et al. Concomitant cholecystectomy during laparoscopic Roux-en-Y gastric bypass in obese patients is not justified: a meta-analysis. Obes Surg 2013; 23: 397-407

[21] Nagem RG, Lazaro-da-Silva A, de Oliveira RM et al. Gallstone-related complications after Roux-en-Y gastric bypass: a prospective study. Hepatobiliary Pancreat Dis Int 2012; 11: 630-635

[22] Shah RJ, Smolkin M, Yen R et al. A multicenter, U. S. experience of single-balloon, double-balloon, and rotational overtube-assisted enteroscopy ERCP in patients with surgically altered pancreaticobiliary anatomy (with video). Gastrointest Endosc 2013; 77: 593-600

[23] Ceppa FA, Gagne DJ, Papasavas PK et al. Laparoscopic transgastric endoscopy after Roux-en-Y gastric bypass. Surg Obes Relat Dis 2007; 3: $21-24$

[24] Falcao M, Campos JM, Galvao NetoM et al. Transgastric endoscopic retrograde cholangiopancreatography for the management of biliary tract disease after Roux-en-Y gastric bypass treatment for obesity. Obes Surg 2012; 22: 872-876

[25] Schreiner MA, Chang L, Gluck M et al. Laparoscopy-assisted versus balloon enteroscopy-assisted ERCP in bariatric post-Roux-en-Y gastric bypass patients. Gastrointest Endosc 2012; 75: 748 - 756

[26] James PD, Kaplan GG, Myers RP et al. Decreasing mortality from acute biliary diseases that require endoscopic retrograde cholangiopancreatography: a nationwide cohort study. Clin Gastroenterol Hepatol 2014; 12: $1151-1159$

[27] Khashab MA, Tariq A, Tariq U et al. Delayed and unsuccessful endoscopic retrograde cholangiopancreatography are associated with worse outcomes in patients with acute cholangitis. Clin Gastroenterol Hepatol 2012; 10: $1157-1161$

[28] Stevenson EK, Rubenstein AR, Radin GT et al. Two decades of mortality trends among patients with severe sepsis: a comparative meta-analysis*. Crit Care Med 2014; 42: 625-631

[29] Kaukonen KM, Bailey M, Suzuki S et al. Mortality related to severe sepsis and septic shock among critically ill patients in Australia and New Zealand, 2000-2012. JAMA 2014; 311: 1308-1316

[30] Folsch UR, Nitsche R, Ludtke R et al. Early ERCP and papillotomy compared with conservative treatment for acute biliary pancreatitis. The German Study Group on Acute Biliary Pancreatitis. N Engl J Med 1997; 336: $237-242$

[31] Tse F, Yuan Y. Early routine endoscopic retrograde cholangiopancreatography strategy versus early conservative management strategy in 
acute gallstone pancreatitis. Cochrane Database Syst Rev 2012; 5: CD009779

[32] Behrns KE, Ashley SW, Hunter JG et al. Early ERCP for gallstone pancreatitis: for whom and when? J J Gastrointest Surg 2008; 12: 629633
[33] Melmer A, Sturm W, Kuhnert B et al. Incidence of Gallstone Formation and Cholecystectomy 10 Years After Bariatric Surgery. Obes Surg 2015; 25: $1171-1176$

[34] Hussan H, Stanich PP, Gray DM et al. Prior bariatric surgery is linked to improved colorectal cancer surgery outcomes and costs: A propensity-matched analysis. Obes Surg 2017; 27: 1047-1055 
- Appendix 1 ICD-9-CM codes used for data extraction and analysis from the Nationwide Inpatient Sample (2007 - 2011).

\begin{tabular}{|c|c|c|}
\hline Diagnosis & ICD-9-CM codes used & Variable location \\
\hline Acute pancreatitis & 577.0 & DX1 \\
\hline History of bariatric surgery & V45.86 & DX2-DX25 \\
\hline Morbid obesity & $278.01, \mathrm{~V} 85.4, \mathrm{~V} 85.41, \mathrm{~V} 85.42, \mathrm{~V} 85.43, \mathrm{~V} 85.44$ & $\mathrm{DX} 2-\mathrm{D} \times 25$ \\
\hline Cholelithiasis or choledocholithiasis (gallstone related) & $\begin{array}{l}574,574.00,574.01,574.10,574.11,574.20,574.21 \\
574.30,574.40,574.41,574.50,574.51,574.60,574.61 \\
574.70,574.71,574.80,574.81,574.90,574.91\end{array}$ & DX2-DX25 \\
\hline Cholangitis & 576.1 & $\mathrm{D} \times 2-\mathrm{D} \times 25$ \\
\hline $\begin{array}{l}\text { Other diseases and obstruction OF BILE DUCT } \\
\text { Adhesions of bile duct [any] } \\
\text { Atrophy of bile duct [any] } \\
\text { Cyst of bile duct [any] } \\
\text { Hypertrophy of bile duct [any] } \\
\text { Stasis of bile duct [any] } \\
\text { Ulcer of bile duct [any] } \\
\text { Bile duct obstruction and jaundice NOS } \\
5762 \text { (bile duct obstruction), } 5769 \text { (disease of the bile } \\
\text { duct), } 7824 \text { (biliary atresia) }\end{array}$ & $576.2,576.8,782.4,576.9$ & DX2-DX25 \\
\hline Pancreatic neoplasm & $156.2,157,157.0,157.1,157.2,157.3,157.8,157.9$ & DX2-DX25 \\
\hline Alcohol related & $\begin{array}{l}\text { 291.0, 291.1, 291.2, 291.3, 291.4, 291.5, 291.81, 291.82, } \\
\text { 291.89, 291.9, 303.00, 303.01, 303.02, 303.03, 303.90, } \\
\text { 303.91, 303.92, 303.93, 305.00, 305.01, 305.02, 305.03, } \\
760.71,980.0,357.5,425.5,535.30,535.31,571.0,571.1 \\
571.2,571.3\end{array}$ & DX2-DX25. \\
\hline History of chronic pancreatitis & 577.1 & DX2-DX25 \\
\hline \multicolumn{3}{|l|}{ TREATMENT } \\
\hline Cholecystectomy & $51.21,51.22,51.23,51.24$ & PR1-PR15 \\
\hline Any ERCP & $\begin{array}{l}51.83,51.84,51.85,51.86,51.87,51.88-51.88,51.10 \\
51.11,51.14,52.13,52.93,52.94,52.98,97.05\end{array}$ & PR1-PR15 \\
\hline Percutaneous biliary procedures & $51.01,51.96,51.98$ & PR1-PR15 \\
\hline Open biliary procedures (common bile duct exploration) & $\begin{array}{l}51.02,51.03,51.04,51.32,51.36,51.37,51.39,51.41 \\
51.43,51.51,51.59,51.63,51.64,51.69,51.71,51.79\end{array}$ & PR1-PR15 \\
\hline Respiratory intubation and mechanical ventilation & $93.90,96.01,96.02,96.03,96.04,96.05,96.70,96.71,96.72$ & PR1 to PR15 \\
\hline Alcohol detoxification/rehabilitation & $94.61,94.62,94.63,94.64,94.65,94.66,94.67,94.68,94.69$ & PR1-PR15 \\
\hline \multicolumn{3}{|l|}{ OUTCOME } \\
\hline Acute respiratory failure & $518.0,518.81,518.82,518.84$ & DX2-DX25 \\
\hline Acute kidney injury & $584.5,584.6,584.7,584.8,584.9,586$ & DX2-DX25 \\
\hline Pancreatectomy & $\begin{array}{l}52.01,52.09,52.22,52.51,52.52,52.59,52.6,52.7,52.95 \\
52.96,52.99\end{array}$ & \\
\hline Roux-en-Y (open and laparoscopic) & $4438,4439,4431$ & PR1 \\
\hline Laparascopic gastric band & 4495 & PR1 \\
\hline Sleeve gastrectomy & $4389,4468,4382$ & PR1 \\
\hline Cholecystectomy & $5121,5122,5123,5124$ & PR2-PR15 \\
\hline
\end{tabular}


- Appendix 2 Summary of missing data for demographic and hospital characteristics in the present analysis of Nationwide Inpatient Sample from $2007-2011$ for 7,343 patients with a history of BRS requiring biliary intervention.

\begin{tabular}{|l|l|}
\hline Total: $\mathbf{7 , 3 4 3}$ & Percent missing \\
\hline Gender & $0 \%$ \\
\hline Race & $9.36 \%$ \\
\hline Income & $1.87 \%$ \\
\hline Type of insurance & $0 \%$ \\
\hline Hospital location & $1.20 \%$ \\
\hline Teaching status & $1.20 \%$ \\
\hline Hospital size & $1.20 \%$ \\
\hline Hospital region & $0 \%$ \\
\hline Admission day & $0 \%$ \\
\hline
\end{tabular}
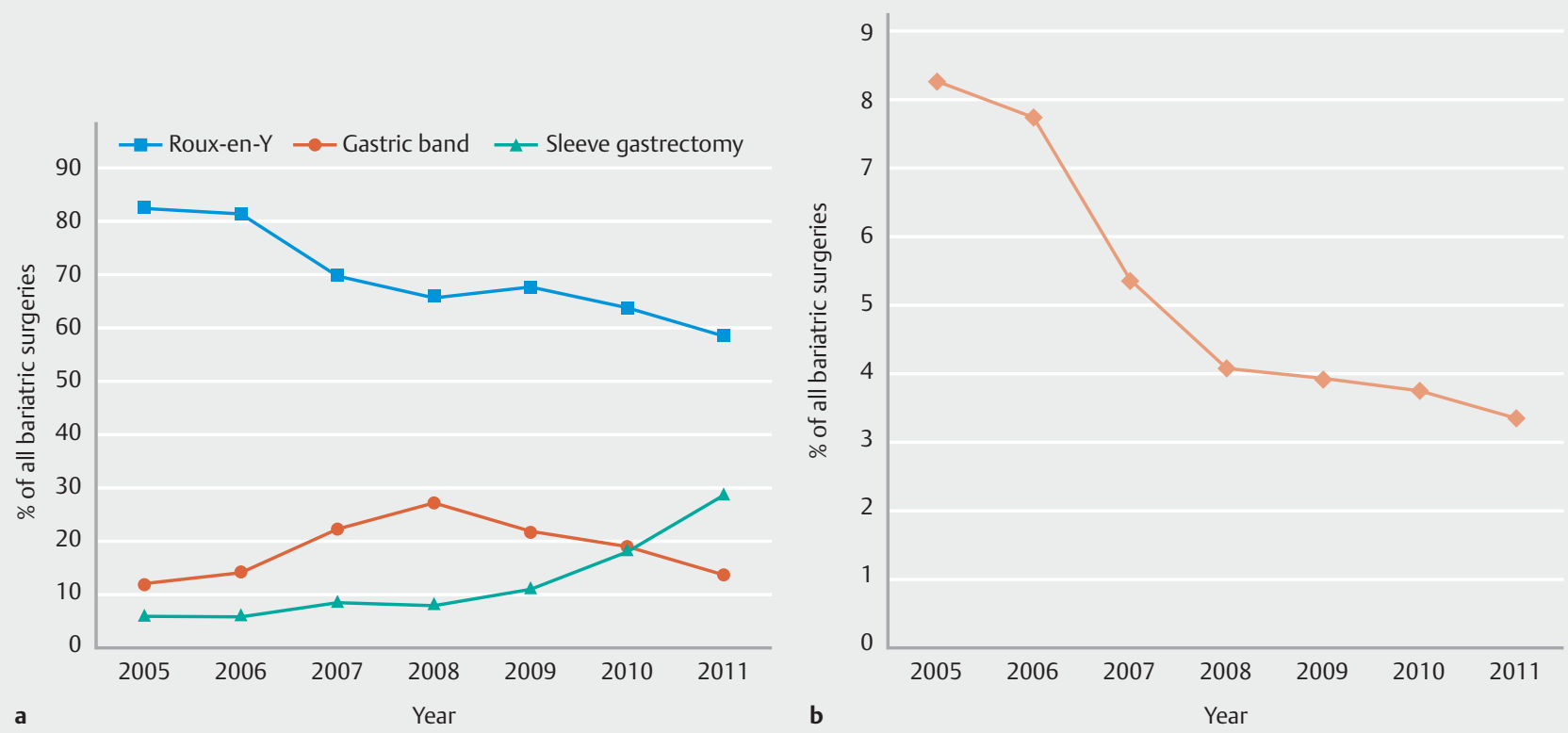

- Appendix 3 Trends in the Type of Bariatric Surgery (a) and Frequency of Concomitant Cholecystectomies (b) in the Nationwide Inpatient Sample from 2005-2011. 
- Appendix 4 Demographics, etiological associations, and outcomes of patients with a history of bariatric surgery undergoing endoscopic or nonendoscopic biliary intervention: Comparison of endoscopic versus PTC versus CBDE.

\begin{tabular}{|c|c|c|c|c|c|c|}
\hline \multirow{2}{*}{$\begin{array}{l}\text { Total: } 7,343 \\
\text { Age (mean, SD) }\end{array}$} & \multicolumn{2}{|c|}{$\begin{array}{l}\text { Endoscopic } \\
n=4,482(\%)\end{array}$} & \multicolumn{2}{|c|}{$\begin{array}{l}\text { PTC } \\
n=1,692(\%)\end{array}$} & \multicolumn{2}{|c|}{$\begin{array}{l}\text { CBDE } \\
n=1,169(\%)\end{array}$} \\
\hline & 50.11 & 30.76 & 51.54 & 27.26 & 53.01 & 24.62 \\
\hline \multicolumn{7}{|l|}{ Gender } \\
\hline - Male & 728 & $16.25 \%$ & 394 & $23.30 \%$ & 311 & $26.62 \%$ \\
\hline - Female & 3,754 & $83.75 \%$ & 1,298 & $76.70 \%$ & 858 & $73.38 \%$ \\
\hline \multicolumn{7}{|l|}{ Race } \\
\hline - White & 3,131 & $76.85 \%$ & 1,225 & $81.46 \%$ & 880 & $81.50 \%$ \\
\hline - Black & 402 & $9.86 \%$ & 147 & $9.78 \%$ & 105 & $9.72 \%$ \\
\hline - Hispanic & 396 & $9.71 \%$ & 102 & $6.80 \%$ & 65 & $5.98 \%$ \\
\hline - Other & 146 & $3.58 \%$ & 29 & $1.96 \%$ & 30 & $2.80 \%$ \\
\hline \multicolumn{7}{|c|}{ Income (national quartile) } \\
\hline . 1 & 948 & $21.48 \%$ & 362 & $22.00 \%$ & 199 & $17.41 \%$ \\
\hline .2 & 1,145 & $25.95 \%$ & 480 & $29.16 \%$ & 285 & $24.94 \%$ \\
\hline .3 & 1,131 & $25.63 \%$ & 440 & $26.73 \%$ & 353 & $30.83 \%$ \\
\hline .4 & 1,189 & $26.95 \%$ & 364 & $22.11 \%$ & 307 & $26.82 \%$ \\
\hline \multicolumn{7}{|l|}{ Type of insurance } \\
\hline - Medicare & 1,129 & $25.19 \%$ & 497 & $29.39 \%$ & 336 & $28.70 \%$ \\
\hline - Medicaid & 297 & $6.62 \%$ & 80 & $4.74 \%$ & 55 & $4.74 \%$ \\
\hline - Private & 2,620 & $58.45 \%$ & 974 & $57.56 \%$ & 672 & $57.49 \%$ \\
\hline - Other & 436 & $9.74 \%$ & 141 & $8.31 \%$ & 106 & $9.07 \%$ \\
\hline \multicolumn{7}{|l|}{ Hospital location } \\
\hline - Rural & 167 & $3.78 \%$ & 136 & $8.15 \%$ & 27 & $2.27 \%$ \\
\hline - Urban & 4,243 & $96.22 \%$ & 1,536 & $91.85 \%$ & 1,143 & $97.73 \%$ \\
\hline \multicolumn{7}{|c|}{ Hospital teaching status } \\
\hline - Nonteaching & 1,835 & $41.61 \%$ & 902 & $53.93 \%$ & 495 & $42.30 \%$ \\
\hline - Teaching & 2,575 & $58.39 \%$ & 770 & $46.07 \%$ & 675 & $57.70 \%$ \\
\hline \multicolumn{7}{|l|}{ Hospital size } \\
\hline - Small & 386 & $8.75 \%$ & 169 & $10.12 \%$ & 82 & $7.05 \%$ \\
\hline - Medium & 898 & $20.36 \%$ & 385 & $23.03 \%$ & 236 & $20.21 \%$ \\
\hline - Large & 3,126 & $70.88 \%$ & 1,118 & $66.85 \%$ & 851 & $72.74 \%$ \\
\hline \multicolumn{7}{|l|}{ Hospital region } \\
\hline - Northeast & 942 & $21.02 \%$ & 313 & $18.48 \%$ & 222 & $18.98 \%$ \\
\hline - Midwest & 1,087 & $24.25 \%$ & 324 & $19.14 \%$ & 216 & $18.50 \%$ \\
\hline " South & 1,321 & $29.48 \%$ & 644 & $38.05 \%$ & 414 & $35.38 \%$ \\
\hline - West & 1,132 & $25.25 \%$ & 412 & $24.33 \%$ & 317 & $27.14 \%$ \\
\hline \multicolumn{7}{|c|}{ Weekend admission } \\
\hline - No & 3,717 & $82.94 \%$ & 1,400 & $82.74 \%$ & 931 & $79.61 \%$ \\
\hline - Yes & 765 & $17.06 \%$ & 292 & $17.26 \%$ & 238 & $20.39 \%$ \\
\hline
\end{tabular}


-Appendix 4 (Continuation)

\begin{tabular}{|c|c|c|c|c|c|c|}
\hline Total: 7,343 & \multicolumn{2}{|c|}{$\begin{array}{l}\text { Endoscopic } \\
n=4,482(\%)\end{array}$} & \multicolumn{2}{|c|}{$\begin{array}{l}\text { PTC } \\
n=1,692(\%)\end{array}$} & \multicolumn{2}{|c|}{$\begin{array}{l}\text { CBDE } \\
n=1,169(\%)\end{array}$} \\
\hline \multicolumn{7}{|l|}{ Elixhauser comorbidity Index } \\
\hline . $<3$ & 2,860 & $63.82 \%$ & 994 & $58.75 \%$ & 514 & $43.96 \%$ \\
\hline - $\geq 3$ & 1,622 & $36.18 \%$ & 698 & $41.25 \%$ & 655 & $56.04 \%$ \\
\hline \multicolumn{7}{|l|}{ ETIOLOGY } \\
\hline Gallstone related & 2,146 & $47.88 \%$ & 854 & $50.45 \%$ & 279 & $23.82 \%$ \\
\hline Pancreaticobiliary Neoplasm & 81 & $1.80 \%$ & 72 & $4.28 \%$ & 125 & $10.67 \%$ \\
\hline Disease of bile duct & 223 & $4.97 \%$ & 40 & $2.38 \%$ & 55 & $4.74 \%$ \\
\hline Bile leak, bile duct injury, biliary peritonitis & 253 & $5.63 \%$ & 152 & $9.01 \%$ & 157 & $13.42 \%$ \\
\hline Bile duct obstruction and jaundice NOS & 262 & $5.84 \%$ & 94 & $5.54 \%$ & 93 & $7.92 \%$ \\
\hline Stent related (changes, others) & 134 & $2.99 \%$ & 21 & $1.26 \%$ & 32 & $2.74 \%$ \\
\hline Chronic Pancreatitis & 54 & $1.21 \%$ & $\mathrm{a}$ & $0.00 \%$ & $\mathrm{a}$ & $0.00 \%$ \\
\hline Sphincter of Oddi dysfunction & 22 & $0.50 \%$ & a & $0.19 \%$ & $\mathrm{a}$ & $0.00 \%$ \\
\hline Abdominal pain & 41 & $0.92 \%$ & a & $0.00 \%$ & a & $0.00 \%$ \\
\hline \multicolumn{7}{|l|}{ Time to PTC/CBDE or ERCP } \\
\hline - $<0$ to 1 day & 2,294 & $51.19 \%$ & 1,672 & $98.81 \%$ & 1,165 & $99.60 \%$ \\
\hline - $2-4$ days & 1,593 & $35.53 \%$ & $\mathrm{a}$ & $0.60 \%$ & $a$ & $0.40 \%$ \\
\hline - 5-10 days & 595 & $13.27 \%$ & $\mathrm{a}$ & $0.59 \%$ & $\mathrm{a}$ & $0.00 \%$ \\
\hline Cholecystectomy & 1,697 & $37.86 \%$ & 1,018 & $60.19 \%$ & 116 & $9.95 \%$ \\
\hline ASSOCIATED DIAGNOSES & \multicolumn{6}{|c|}{ (DX1-DX25) } \\
\hline Sepsis & 184 & $4.10 \%$ & 132 & $7.80 \%$ & 171 & $14.60 \%$ \\
\hline Acute Pancreatitis & 1,146 & $25.57 \%$ & 198 & $11.70 \%$ & 162 & $13.84 \%$ \\
\hline Cholangitis & 532 & $11.87 \%$ & 210 & $12.40 \%$ & 178 & $15.19 \%$ \\
\hline \multicolumn{7}{|l|}{ OUTCOME } \\
\hline Death & $\mathrm{a}$ & $0.22 \%$ & $\mathrm{a}$ & $0.30 \%$ & 15 & $1.26 \%$ \\
\hline Length of stay $\geq 7$ days & 1,215 & $27.12 \%$ & 783 & $46.27 \%$ & 569 & $48.66 \%$ \\
\hline Length of stay & 5.31 & 9.46 & 7.11 & 9.89 & 7.83 & 13.89 \\
\hline Total charges & 50,664 & 92,279 & 66,873 & 137,658 & 60,624 & 106,082 \\
\hline
\end{tabular}


- Appendix 5 Multivariate linear regression model for healthcare utilization in patients with a history of bariatric surgery undergoing biliary intervention with the addition of time period as a variable, Nationwide Inpatient Sample, 2007 - 2011.

\begin{tabular}{|c|c|c|c|c|c|c|}
\hline & \multicolumn{3}{|c|}{ Length of stay } & \multicolumn{3}{|c|}{ Total charges } \\
\hline & Days & $95 \% \mathrm{Cl}$ & $P$-value & $\$$ & $95 \% \mathrm{Cl}$ & $P$-value \\
\hline Primary procedure & & & $<0.0001$ & & & $<0.0001$ \\
\hline - ERCP & Reference & & & Reference & & \\
\hline - CBDE/PTC & 1.77 & $(1.32,2.22)$ & & 11,885 & $(6,462,17,308)$ & \\
\hline Age & 0.01 & $(-0.01,0.02)$ & 0.4191 & -78 & $(-224,67)$ & 0.2912 \\
\hline Gender & & & 0.7549 & & & 0.0409 \\
\hline - Male & Reference & & & Reference & & \\
\hline - Female & -0.08 & $(-0.60,0.43)$ & & $-6,034$ & $(-11,818,-251)$ & \\
\hline Hospital location & & & 0.0365 & & & $<0.0001$ \\
\hline - Rural & Reference & & & Reference & & \\
\hline - Urban & 0.64 & $(0.04,1.23)$ & & 19,313 & $(13,710,24,917)$ & \\
\hline Hospital teaching status & & & 0.9254 & & & 0.6763 \\
\hline - Nonteaching & Reference & & & Reference & & \\
\hline - Teaching & 0.03 & $(-0.51,0.56)$ & & $-1,409$ & $(-8,035,5,217)$ & \\
\hline Hospital region & & & 0.8802 & & & $<0.0001$ \\
\hline - Northeast & Reference & & & Reference & & \\
\hline - West & -0.17 & $(-0.86,0.53)$ & & 14,640 & $(4,446,24,834)$ & \\
\hline - South & 0.04 & $(-0.59,0.67)$ & & $-2,360$ & $(-11,336,6,615)$ & \\
\hline - Midwest & 0.09 & $(-0.55,0.73)$ & & $-7,003$ & $(-16,173,2,167)$ & \\
\hline Elixhauser comorbidity Index & & & $<0.0001$ & & & $<0.0001$ \\
\hline - $<3$ & Reference & & & Reference & & \\
\hline - $\geq 3$ & 1.27 & $(0.79,1.75)$ & & 10,306 & $(5,330,15,282)$ & \\
\hline Gallstone related & -0.79 & $(-1.22,-0.36)$ & 0.0004 & $-3,731$ & $(-8,624,1,162)$ & 0.1348 \\
\hline Pancreaticobiliary neoplasm & 1.55 & $(0.03,3.08)$ & 0.0459 & 4,032 & $(-7,632,15,697)$ & 0.4974 \\
\hline $\begin{array}{l}\text { Bile leak, bile duct injury, biliary } \\
\text { peritonitis }\end{array}$ & 0.79 & $(-0.38,1.96)$ & 0.1867 & 5,770 & $(-6,532,18,072)$ & 0.3572 \\
\hline Chronic pancreatitis & -1.34 & $(-2.29,-0.38)$ & 0.0064 & $-17,281$ & $(-35,898,1,336)$ & 0.0688 \\
\hline Time to PTC/CBDE or ERCP & & & $<0.0001$ & & & $<0.0001$ \\
\hline - <0 to 1 day & Reference & & & Reference & & \\
\hline - 2-4 days & 1.72 & $(1.29,2.15)$ & & 12,112 & $(7,779,16,445)$ & \\
\hline - 5-10 days & 6.29 & $(5.49,7.09)$ & & 43,714 & $(33,387,54,040)$ & \\
\hline Time period & & & 0.7669 & & & 0.0227 \\
\hline - 2007-2008 & Reference & & & & & \\
\hline - 2009-2011 & 0.07 & $(-0.37,0.50)$ & & 6,378 & $(893,11,863)$ & \\
\hline
\end{tabular}


- Appendix 6 Multivariate linear regression model for healthcare utilization in patients with a history of bariatric surgery undergoing endoscopic biliary intervention with the addition of time period as a variable, Nationwide Inpatient Sample, 2007 - 2011.

\begin{tabular}{|c|c|c|c|c|c|c|}
\hline & \multicolumn{3}{|c|}{ Length of stay } & \multicolumn{3}{|c|}{ Total charges } \\
\hline & Days & $95 \% \mathrm{Cl}$ & $P$-value & $\$$ & $95 \% \mathrm{Cl}$ & $P$-value \\
\hline Endoscopic intervention & & & $<0.0001$ & & & 0.0078 \\
\hline - Successful & Reference & & & Reference & & \\
\hline - Failed & 2.18 & $(1.16,3.19)$ & & 14,400 & $(3,819,24,981)$ & \\
\hline Age & 0.01 & $(-0.01,0.03)$ & 0.2081 & 145 & $(-21,311)$ & 0.0858 \\
\hline Race & & & 0.7097 & & & 0.1255 \\
\hline - White & Reference & & & Reference & & \\
\hline - Black & -0.01 & $(-0.75,0.73)$ & & 4,778 & $(-4,873,14,430)$ & \\
\hline - Hispanic & -0.30 & $(-0.96,0.36)$ & & 8,178 & $(1,249,15,107)$ & \\
\hline - Other & 0.37 & $(-0.91,1.66)$ & & -860 & $(-14,039,12,319)$ & \\
\hline Hospital size & & & 0.1669 & & & 0.3426 \\
\hline - Small & Reference & & & Reference & & \\
\hline - Medium & 0.12 & $(-0.49,0.73)$ & & 3,299 & $(-7,999,14,597)$ & \\
\hline - Large & 0.49 & $(-0.07,1.05)$ & & 6,217 & $(-2,439,14,873)$ & \\
\hline Chronic pancreatitis & -0.14 & $(-0.80,0.52)$ & 0.6731 & $-12,795$ & $(-34,539,8,950)$ & 0.2479 \\
\hline Time to ERCP & & & $<0.0001$ & & & $<0.0001$ \\
\hline - $<0$ to 1 day & Reference & & & Reference & & \\
\hline - $2-4$ days & 1.87 & $(1.41,2.33)$ & & 14,894 & $(9,663,20,124)$ & \\
\hline - 5-10 days & 7.16 & $(6.09,8.22)$ & & 38,859 & $(28,422,49,296)$ & \\
\hline Time period & & & 0.4483 & & & 0.0596 \\
\hline - 2007-2008 & Reference & & & & & \\
\hline . 2009-2011 & 0.18 & $(-0.29,0.65)$ & & 5,950 & $(-242,12,142)$ & \\
\hline
\end{tabular}

